\title{
Advanced Head and Neck Carcinoma
}

National Cancer Institute

\section{Source}

National Cancer Institute. Advanced Head and Neck Carcinoma. NCI Thesaurus. Code C129861.

A head and neck carcinoma that has spread extensively to other anatomical sites or is no longer responding to treatment. 